\title{
Millimeter wave-induced changes in membrane properties of leech Retzius neurons
}

Victor Pikov ${ }^{1,2 *}$, Peter H. Siegel ${ }^{3,4}$

1 Neural Engineering Program, Huntington Medial Research Institutes, Pasadena, CA

1 Department of Ophthalmology, University of Southern California, Los Angeles, CA

2 Division of Biology, California Institute of Technology, Pasadena, CA

3 Division of Engineering and Applied Sciences, California Institute of Technology, Pasadena, CA

* Corresponding author

\begin{abstract}
This study evaluated a novel method for modulation of neuronal excitability using non-invasive delivery of millimeter waves. Millimeter waves at $60 \mathrm{GHz}$ and incident power density of 100-600 $\mu \mathrm{W} / \mathrm{cm}^{2}$ were applied to three intact segmental ganglia of the adult leach, and intracellular neuronal activity was recorded from the Retzius neurons using intracellular glass electrode. Transient dosedependent increase in the plasma membrane permeability was observed. In addition, in one of the examined neurons, a decrease in the neuronal firing rate was also evident. The results provide strong evidence for the feasibility of modulating neuronal excitability using non-invasive delivery of millimeter waves, and will be explored further for applications in basic neuroscience and treatment of neurological disorders.
\end{abstract}

\section{Introduction}

Epidural electrical stimulation (EES) can be used modulation of neuronal excitability and activity in the brain. However, the vertical localization of EES is limited due to considerable lateral current spread through cerebrospinal fluid-filled space at the brain surface (Wongsarnpigoon and Grill, 2008). This study attempts to develop a novel method for modulation of neuronal excitability using non-invasive delivery of millimeter waves (MMWs). Such mode of neuromodulation may be important for different brain regions that exhibit columnar topographical organization. A prime example of such organization is the cerebral cortex, where functional processing units ("microcircuits") are organized vertically through an intricate inter-laminal connectivity (Haeusler and Maass, 2007). Due to their long wavelength as compared to visible and infrared light, MMWs produce negligible scattering in tissue (Bashkatov et al., 2005) providing better depth penetration in the tissue. We estimate that MMWs provide the highest ratio of vertical to horizontal energy spread on a millimeter scale compared to existing neuronal modulation methods.

In this study, the effects of MMWs on neurons will be evaluated in the segmental ganglion of the adult leach. The leech ganglion provides an attractive model for evaluation of intracellular neuronal activity due to its easy dissection and ability to mount an intact ganglion in the Petri dish coated with a layer of paraffin. The MMWs can be readily transmitted from below the dish, taking advantage of the paraffin transparence in this frequency range and thus mostly avoiding MMW absorption by the saline solution in the dish. Known distance of the ganglion from the MMW waveguide port and absence of saline in the MMW path allow rather accurate estimation of the incident power density at the plane of the Retzius neurons. Furthermore, immediate absorption of the MMWs by the ganglion allows maintaining

Photonic Therapeutics and Diagnostics VII, edited by N. Kollias, et al., Proc. of SPIE Vol. 7883, $788356 \cdot$ ? 2011 SPIE · CCC code: $1605-7422 / 11 / \$ 18 \cdot$ doi: $10.1117 / 12.875758$ 
the saline in the dish at a constant temperature, thus avoiding indirect effects of MMWs on neurons via heating of the medium. In summary, the ex vivo preparation of the segmental leech ganglion provides an attractive animal model to evaluate the neuronal responses during MMW exposure.

\section{Methods}

Adult 4-6 g adult leeches (Hirudo medicinalis) were obtained from a commercial supplier. The animals were kept in aquarium in artificial pond water $(1: 1000$ SeaWater $)$ at $20^{\circ} \mathrm{C}$. The animals were not fed for at least 1 month prior to dissection. The leech was stretched longitudinally and pinned down dorsal side up on the Silgard-coated dissection box filled with the ice-cold leech saline $\left(+4^{\circ} \mathrm{C}\right)$ to anesthetize the animal. Medial longitudinal incision was made and the blood was washed away. Connective tissue and blood vessel overlaying the ganglion chain were incised. The vessel was also cut longitudinally on the ventral side to create "handles" for immobilizing the lateral roots. To reduce variability, we always used the segments 7-10 of the 21 midbody segments.

A single ganglion was isolated and pinned with ventral surface up in a small Petri dish bottomcoated with paraffin and filled with a leech saline. The rostral and caudal connectives as well as the "handles" surrounding the lateral roots were secured with six stainless steel pins $(1 \mathrm{~mm}$ long, $50 \mu \mathrm{m}$ in diameter). The ventral orientation was established by observing the dorsal-ward curvature of the lateral roots. The Petri dish was placed on the upright Olympus microscope BX-FM. The Retzius neurons were

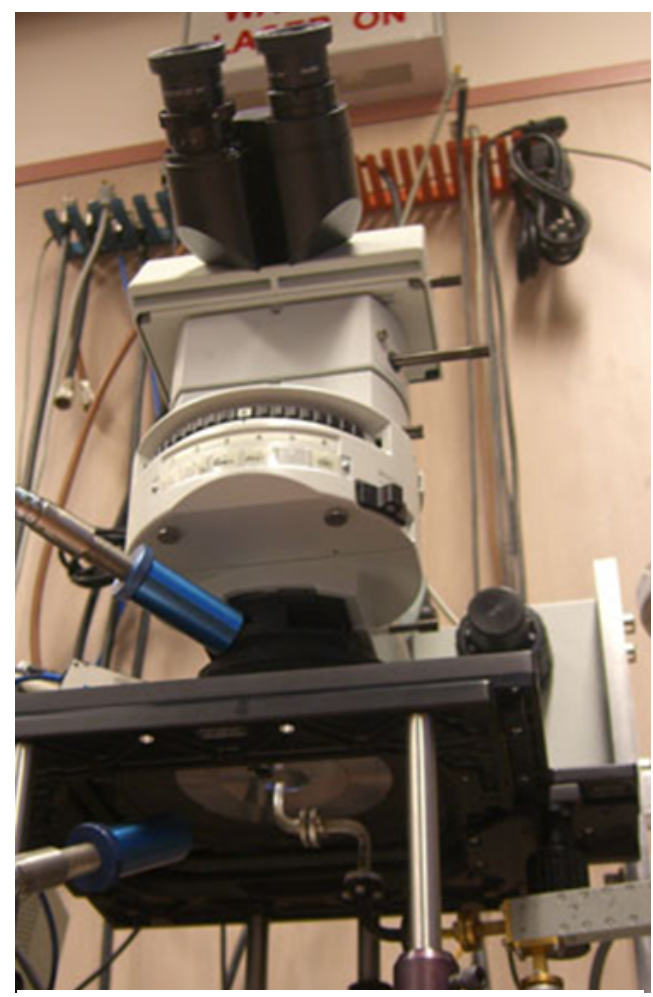

Figure 1.Experimental setup. identified by their size $(50-80 \mu \mathrm{m})$ and central location in the ganglion. The intracellular neuronal activity in the Retzius neurons was penetrated with glass intracellular electrodes filled with potassium acetate ( $3 \mathrm{M}, 20-30 \mathrm{M} \Omega$ ). Currentclamp recordings obtained using a an intracellular amplifier AxoClamp-2A, digitizer Digidata 1200, and a computer running pCLAMP 9 software (all from Molecular Devices, Sunnyvale, CA). Data records were digitized at $10 \mathrm{kHz}$. Intracellular depolarizing current was held continuously at $+500 \mathrm{pA}$. Changes in the input resistance were calculated by dividing the change in the resting membrane potential by the amplitude of the injected depolarizing current: $\Delta R_{n}=\Delta V / I$.

The MMW exposure system consisted of a custom assembled MMW source (injection-locked IMPATT oscillator) operating at $60.125 \mathrm{GHz}$ and producing up to 185 $\mathrm{mW}$ of continuous wave $(\mathrm{CW})$ power at the exit of the openended rectangular waveguide (WR-153, aperture $8 \times 1.9 \mathrm{~mm}$ ). The waveguide was carefully positioned below the Petri dish and MMWs were applied from a distance of $10 \mathrm{~mm}$ (Figure 1). Power was continually monitored through a directional coupler and calibrated RF power meter (HP 436A, Agilent Technologies, Santa Clara, CA). The linearly polarized MMW fields exiting the rectangular waveguide expanded at a half angle of $\sim 27^{\circ}$ in the $\mathrm{H}$ plane (parallel to the wide wall) and $56^{\circ}$ in the E plane (along the short wall). Using published data for the complex permittivity of water and water-based media at 60 $\mathrm{GHz}$ (Zhadobov et al., 2008), we calculated that $\sim 45 \%$ of power was reflected upon contact with the 
fluid surface, leaving $55 \%$ of available power for absorption by the ganglion. Using the published data for the same frequency and similar ionic media (Zhadobov et al., 2008) and a Beer's law $\left(\mathrm{I}=\mathrm{I}_{0} \mathrm{e}^{-\mathrm{ax}}\right)$ for the intensity drop with penetration depth $\mathrm{x}$ (in $\mathrm{cm}$ ), we estimated the loss tangent for MMW absorption by the ganglion and leech saline to be 1.48. This translates into the absorption coefficient of $52 \mathrm{~cm}^{-1}$, indicating the power drop of $99.5 \%$ in $1 \mathrm{~mm}$. The estimated distance between the ventral surface of the ganglion, containing the Retzius neurons, and the bottom of the dish was $400 \mu \mathrm{m}$. Therefore, the calculated incident power density (IPD) of MMWs at the level of Retzius neurons was in the range of 100 to $600 \mu \mathrm{W} / \mathrm{cm}^{2}$, which is below the US-approved maximal permissible exposure of $1 \mathrm{~mW} / \mathrm{cm}^{2}$ (Chou and D'Andrea, 2005). During MMW exposure, temperature of saline in the dish was monitored using a thermocouple probe positioned near the bottom of the dish $3 \mathrm{~cm}$ away from the center. The probe was connected to a TC2BIP temperature controller with $0.1^{\circ} \mathrm{C}$ accuracy and digital readout screen (Cell MicroControls, Norfolk, VA).

\section{Results}

We evaluated the changes in the membrane input resistance $R_{n}$, estimated as the change of the resting membrane potential during the injection of a depolarizing current $(\Delta \mathrm{V} / \mathrm{I})$. This parameter was chosen to provide a measure of membrane permeability that is not dependent on the presence of the action potentials and, thus, not susceptible to confounding variability in the firing rate. Three Retzius neurons were evaluated at three IPDs each. An averaged $R_{n}$ value was calculated for each 10 -sec period during the 60-sec exposure to MMWs (Figure 2). In all three studied neurons, the input resistance has decreased transiently during the exposure, with a quick recovery immediately after the termination of exposure. The magnitude of the effect was proportional to the intensity of applied MMW power.

In addition to the changes in the membrane input resistance, we have also observed the transient slowing down of the firing rate in one of the examined Retzius neurons (n01) (Figure 3). The duration of the effect on the firing rate was the same as the duration of the effect on the input resistance, as can be seen in Figure 3. In other two Retzius neurons, there was no change in the firing rate.

There was no detectable temperature increase in the saline solution during the MMW exposure (data not shown). There was also no change in the amplitude of action potentials in all three studied neurons.

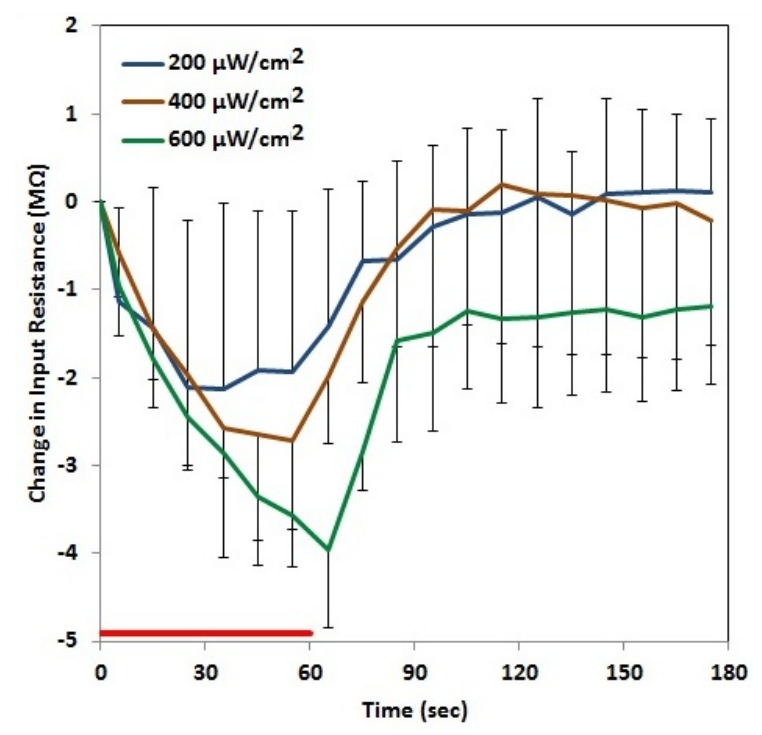

Figure 2. The effect of the three IPD levels of MMWs on the $\Delta R_{n}$. An average value for each time point is presented for three studied Retzius neurons. Error bars represent standard deviations. Red bar indicates the duration of the $M M W$ exposure. 

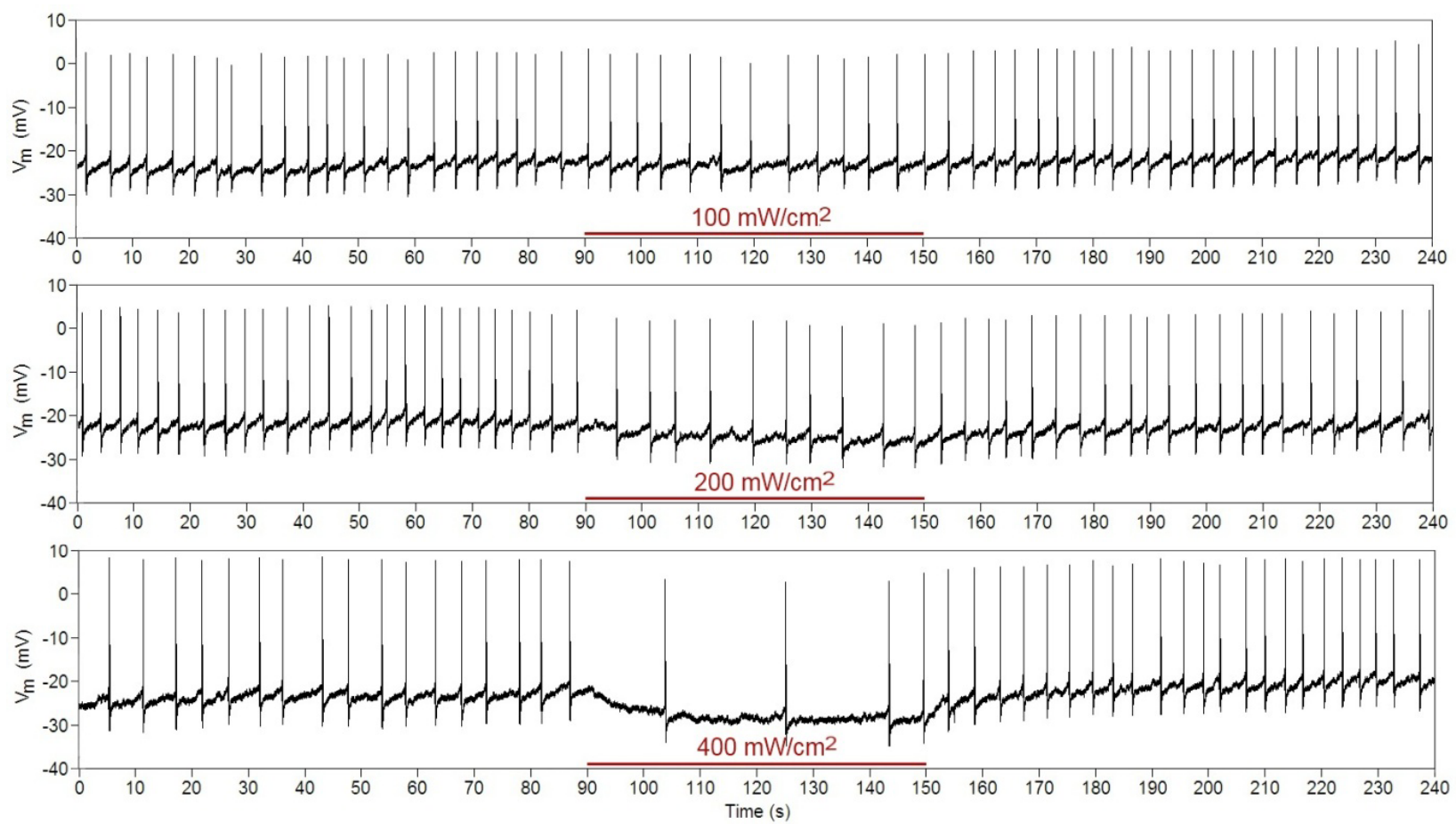

Figure 3. Sample recordings of intracellular voltage $\left(V_{m}\right)$ in the Retzius neuron $n 01$ during three IPD levels of MMW exposure, applied in a random order during the experiment. Red bars indicate the duration of the MMW exposure with the value above the bar indicating the IPD level.

\section{Discussion}

The transient dose-dependent increase in the plasma membrane permeability was observed in three studied Retzius neurons. In one of the neurons, there was a corresponding decrease in the firing rate. These results extend our recent findings from the cortical slices (Pikov et al., 2010) and provide strong evidence for the feasibility of controlling neuronal excitability using non-invasive delivery of MMWs, which can be explored further for applications in basic neuroscience and treatment of neurological disorders.

The mechanisms of MMW action on the neuronal membrane may be broadly attributed to general heating or a specific absorption of MMWs by water, lipids, or proteins in or near the neuronal plasma membrane. There was no temperature change detected in the bath solution, suggesting that heating did not play a major role in the observed reduction of the input resistance. However, the thermal effect cannot be completely excluded as no measurement of temperature was taken directly at the Retzius neuron location. In the future studies, we hope to provide temperature measurements at a single-neuron level by utilizing the recently developed method based on Raman spectroscopy (Pikov and Siegel, 2010).

\section{References}

Bashkatov AN, Genina EA, Kochubey VI, Tuchin VV, "Optical properties of human skin, subcutaneous and mucous tissues in the wavelength range from 400 to $2000 \mathrm{~nm}$," J Physics D: Appl Physics 38, 2543-2555 (2005). 
Chou C-K, D'Andrea J, [IEEE Standard for Safety Levels With Respect to Human Exposure to Radio Frequency Electromagnetic Fields, $3 \mathrm{kHz}$ to $300 \mathrm{GHz}$ (2005).

Haeusler S, Maass W, "A statistical analysis of information-processing properties of lamina-specific cortical microcircuit models," Cereb Cortex 17, 149-162 (2007).

Pikov V, Siegel PH, "Thermal monitoring: Raman spectrometer system for remote measurement of cellular temperature on a microscopic scale," IEEE Eng Med Biol Mag 29,63-71 (2010).

Pikov V, Arakaki X, Harrington M, Fraser SE, Siegel PH, "Modulation of neuronal activity and plasma membrane properties with low-power millimeter waves in organotypic cortical slices," J Neural Eng 7, 045003 (2010).

Wongsarnpigoon A, Grill WM, "Computational modeling of epidural cortical stimulation," J Neural Eng 5, 443-454 (2008).

Zhadobov M, Sauleau R, Le Drean Y, Alekseev SI, Ziskin MC, "Numerical and Experimental Millimeter-Wave Dosimetry for In Vitro Experiments," IEEE Trans Microwave Theory Tech 56, 2998-3007 (2008). 